\title{
New serum biomarker identification and analysis by mass spectrometry in cervical precancerous lesion and acute cervicitis in South China
}

This article was published in the following Dove Press journal: Cancer Management and Research

\author{
Feng Qiu',* \\ Bingsen $\mathrm{Su}^{2} *$ \\ Zhao $\mathrm{Li}^{3}$ \\ Wenke Chen' \\ Longbing Cao' \\ Fu Chen ${ }^{4}$ \\ Dongdong $\mathrm{Liu}^{4}$ \\ Jingling $\mathrm{He}^{5}$ \\ $\mathrm{Ni} \operatorname{Lin}^{3}$
}

\begin{abstract}
'Department of Laboratory Medicine, Nanhai Hospital, Southern Medical University, Foshan, Guangdong 528244, People's Republic of China; ${ }^{2}$ Department of Laboratory Medicine, Zhongshan Torch Development Zone Hospital, Zhongshan, Guangdong 528437, People's Republic of China; ${ }^{3}$ Department of General Practice Center, Nanhai Hospital, Southern Medical University, Foshan, Guangdong 528244, People's Republic of China; ${ }^{4}$ Department of Laboratory Medicine, the Second Affiliated Hospital of Guangzhou University of Chinese Medicine, Guangzhou, Guangdong 510120, People's Republic of China; ${ }^{5}$ Department of Gynecology, the Second Affiliated Hospital of Guangzhou University of Chinese Medicine, Guangzhou,

Guangdong 510120, People's Republic of China
\end{abstract}

*These authors contributed equally to this work

Correspondence: Ni Lin

Department of General Practice Center, Nanhai Hospital, Southern Medical University, 28 Liguan Road, Lishui Town, Nanhai District, Foshan City, Guangdong Province 528244, People's Republic of China

Tel +8607578563 II 24

Email linlinnini@hotmail.com
Background: According to the statistics of WHO/IARC, cervical cancer (CC) has become the fourth malignant cancer of female worldwide and it is one of the main causes of death of women in developing countries.

Purpose: Potential plasma and metabolic biomarkers for $\mathrm{CC}$ precancerous lesions and cervicitis were indicated by LC-MS techniques, and their underlying mechanisms and functions were analyzed.

Methods: Plasma samples were selected from healthy people (control), low-grade squamous intraepithelial lesions (LSIL), high-grade squamous intraepithelial lesions (HSIL), CC, and post-treatment patients. All polypeptide types and sequences were detected by LC-MS/MS and the results were normalized by using Pareto-scaling. Potential metabolic biomarkers were screened by applying MetaboAnalyst 4.0 software and XCMS software, and analysis of variance and enrichment analysis were performed. Metabolic pathway analysis and functional enrichment analysis were used to further investigate the significance and pathological mechanisms of potential biomarkers.

Results: Compared with healthy people, 9 differentially expressed metabolites were screened, 4 of which were up-regulated and 5 were down-regulated. LSIL group screened 7 differentially expressed metabolites, 5 of which were up-regulated and 2 were down-regulated; CC group screened 12 differentially expressed metabolites were screened, of which 9 were up-regulated and 3 were down-regulated. Eight differentially expressed metabolites were screened in the IF group, of which 5 showed up-regulation and 3 showed down-regulation. In functional enrichment analysis, differential metabolism was found to be associated with addition and coagulation cascades. Among all potential biomarkers, 2-amino-3-methyl-1-butanol, L-carnitine, Asn Asn Gln Arg, Ala Cys Ser Trp, Soladulcidine, Ala Ile Gln Arg, 2-amino-3 -Methyl-1-butanol, L-carnitine, Asn Asn Gln Arg, Ala Cys Ser Trp, Soladulcidine, Ala Ile Gln Arg can be used as predictors of precancerous lesions at different stages of CC. Among all biomarkers, $6 \alpha$-fluoro -11ß1,17-dihydroxypren-4-ene-3,20-dione has higher expression in the CC and HSIL groups and lower expression in the treatment group.

Conclusion: By applying molecular markers to assess the progression of the disease, the accuracy and specificity of the diagnosis can be improved, which has certain prospects in clinical applications.

Keywords: cervical precancerous lesion, acute cervicitis, LC-MS, metabonomics, serum biomarkers

\section{Introduction}

According to the statistics of WHO/IARC, cervical cancer (CC) has become the fourth malignant cancer of female worldwide, and it is one of the main causes of death of 
women in developing countries. ${ }^{1}$ In 2015 , the new cases of cervical cancer have reached 98,900 with 30,500 death cases. In the recent 20 years, the morbidity of $\mathrm{CC}$ has been on the rise and the death rate is relatively stable. ${ }^{2}$ Due to the environmental pollution and bad health habits, the patients of $\mathrm{CC}$ are becoming younger with the increasing rate of precancerous lesions. ${ }^{3}$ The squamous intraepithelial lesion (SIL) is one of the common types of precancerous lesions of cervical cancer, which has increased the risk of getting cervical cancer. The chronic cervicitis also has potential risks to become SIL. Therefore, early diagnosis of cervical cancer is essential to improve the survival rate.

Colposcope examination and cervix biopsy are the main clinical diagnosis methods currently. These methods have high dependency on the subjectivity of the medical staff, which will easily cause instability of the accuracy and specificity of the diagnosis. ${ }^{4,5}$ Therefore, research on finding accurate and objective diagnosis indexes to judge the progression of cervical cancer is of great importance. ${ }^{6}$ In recent years, the early screening and diagnosis of different new types of $\mathrm{CC}$ are popular; however, the clinical application is not effective enough. For instance, the specificity of P16/Ki67 chromosome detection of CIN2 and CIN3 is only $58.9 \%$ and $56.9 \%$, respectively; ${ }^{7}$ the detection rates of photoelectric detection system are $74.4 \%$ and $78.8 \%$, respectively,. ${ }^{8}$ Although the accuracy rate of MRI staging diagnosis is $86 \%$, which has obvious advantages in detecting parametrial invasion of cervical carcinoma, the value of diagnosis on IA cervical cancer is limited. ${ }^{9}$ Therefore, the diagnosis accuracy and specificity of precancerous lesions remain low, which are the primary causes of high false positive rate, missing detection and misdiagnosis.

Compared with the common diagnosing method of colposcope examination and cervix biopsy, noninvasive diagnosis realized by molecular marker is of great importance clinically. From a clinical point of view, the term tumor marker applies to all substances produced and released by tumor cells or host cells, the presence of which may be in serum or other biological fluids. Ideal tumor markers should have high sensitivity (SE) and high specificity (SP) to distinguish cancer patients from healthy subjects or benign disease patients and should be secreted into the circulation and activities at a concentration proportional to tumor burden. CA 125 is the most reliable serum marker for ovarian cancer. Although its role in screening for malignant tumors is controversial, serum CA 125 detection is very useful for the differential diagnosis of ovarian masses, especially after menopause, as well as monitoring of chemotherapy response and histologically confirmed follow-up cancer in patients with ovarian cancer. ${ }^{10,11}$ Metabonomics has been widely applied on the biomarker screening of cancerous metabolism to infer the existence and progression of cancer. $^{12}$ The metabolites are the final products of all kinds of bioprocesses and they are likely to become the accurate biomarkers which can reflect the upstream biological events (such as gene mutation and environmental changes). ${ }^{13}$ The changes in metabolites and their pathways can provide a better understanding of the happening and progression of cancer. ${ }^{14}$ Kai conducted UPLC/MS detection of the plasma metabolite of the cervical cancer patients from northern China. By comparing the plasma metabolic profiling of 70 cases of cervical cancer patients and 80 healthy people, Kai found 62 differential metabolites, among which bilirubin, LysoPC (17:0), n-oleoyl threonine, 12-hydroxydodecanoic acid and tetracosahexaenoic acid can serve as the marker of cervical cancer. ${ }^{15}$ Ye applied 1HNMR spectral to analyze the plasma of 22 cases of chronic inflammation, 9 cases of cervical intraepithelial neoplasia (CIN) and 18 cases of cervical cancer patients from Beijing, China. Through multivariate modeling of PLS-DA analysis, it is easy to differentiate cancer group and non-cancer group (chronic cervicitis and CIN). There are significant differences of formate, tyrosine, $\beta$-glucose, inositol, glycine, carnitine, glutamine, acetate, alanine, valine, isoleucine and very-low-density lipoprotein (VLDL) among groups. ${ }^{16}$ Hasim analyzed the plasma metabolic profiling of $\mathrm{CIN}$ and $\mathrm{CC}$ from Xinjiang Region, finding that compared to healthy people, the CIN patients had higher level of VLDL, acetone, unsaturated lipid and carnitine, and the $\mathrm{CC}$ patients had higher level of acetate and formate. Compared to CIN patients, the CC patients had higher level of acetate, formate, lactate, isoleucine, leucine, valine, alanine, glutamine, histidine, tyrosine, acetylcysteine, myo-inositol, glycoprotein, $\alpha$ glucose and $\beta$-glucose. ${ }^{17}$ Qun applied LC-QTOF/MS to analyze the urine metabolic profiling of $\mathrm{CC}$ patients from northern China, finding that compared to healthy people, there was significant difference in 3-methylhistidine, citric acid, cytosine, indoleacetic acid, salicyluric acid, L-methionine, aminomalonic acid, glutaric acid, ursodeoxycholic acid and $\mathrm{N}$-acetylornithine of the $\mathrm{CC}$ patients. The metabolic pathways included citrate cycle, lysine degradation, tryptophan metabolism, cysteine and methionine metabolism, and the changes of these pathways could provide evidence for the happening of early $\mathrm{CC}$, which could be recognized as the potential markers to predict the early happening of CC. ${ }^{18}$

This research intends to use LC-MS/MS technology to study the plasma metabolic profiling of cervical inflammation, LSIL, HSIL CC patients from Guangdong, a southern province of China. By screening the differential metabolism, 
the potential diagnostic marker in different stages of lesions of plasma metabolism is then found out, which is of clinical significance for the screening, diagnosis, detecting accuracy and specificity of precancerous cervical lesions and even the treatment efficacy of cervical cancer.

\section{Materials and methods}

\section{Research object and sampling}

The serum samples were collected from the patients in Gynecology Department of Nanhai Hospital affiliated to Southern Medical University from May 2015 to April 2016. All patients were non-pregnant and not in menstrual period. According to the results of TCT, colposcope and biopsy, the collected samples were divided into groups of cervicitis (HPV positive) (Inflammation, IF) with 10 cases, low grade squamous intraepithelial lesion (LSIL) with 5 cases, high grade squamous intraepithelial lesion (HSIL) with 8 cases, cervical cancer (CC) with 19 cases and cervical cancer treatment (Treatment) with 14 cases. Meanwhile, 11 serum samples from healthy women were also collected as control group (Control, CON). In the control group, the leucorrhea cleanliness was below level II and the samples were all detected negative in HPV, TCT, mycoplasma, chlamydia and gonococcus. This research had passed the investigation of Ethics Committee of Nanhai Hospital affiliated to Southern Medical University (Certificate No. B2016-019-01). The blood samples were collected by anti-coagulation tubes and the centrifugal plasma was kept under $-20^{\circ} \mathrm{C}$ for LC-MS/MS detection. All patients and healthy controls provided written informed consent, and this study was conducted in accordance with the Declaration of Helsinki.

\section{Preconditioning of plasma}

Take $100 \mu \mathrm{L}$ of plasma sample and add $600 \mu \mathrm{L}$ of methanol (containing $1 \mathrm{mM}$ butylhydroxytoluene) was for $5 \mathrm{~min}$ ultrasonic processing. Then, add $1.8 \mathrm{~mL}$ of Methyl Tertbutyl Ether and oscillate at room temperature for 1 hr. Add $500 \mu \mathrm{L}$ of water and incubate for 10 mins at room temperature and oscillate without interruption. After 2-min still standing, subject to $12,000 \mathrm{rpm}$ centrifugation for 10 mins. Pipette $600 \mu \mathrm{L}$ of the upper layer lipid and $300 \mu \mathrm{L}$ of the lower layer $(2: 1)$ aqueous layer and transfer to a new EP tube, vacuum dry, dissolve with $200 \mu \mathrm{L}$ of acetonitrile and water $(1: 1)$, centrifuge to take the supernatant, using mixed layer and aqueous layer metabolite conditions. Set this sample group as $\mathrm{C}$.

\section{Metabolic profiling detection by LC-MS}

The experiment used ACE (Aberdeen, Scotland) Excel2C18 PFP $(100 \times 2.1 \mathrm{~mm}, 2 \mu \mathrm{m})$ chromatographic column and C18 guard column. Mobile phase A contained aqua with $0.1 \%$ formic acid and mobile phase B contained acetonitrile with $0.1 \%$ formic acid. The chromatographic gradient started from 2\% B for $1 \mathrm{~min}$ and entered $98 \% \mathrm{~B}$ in 10 mins. This had kept for $2 \mathrm{~min}$ and then it dropped to $2 \%$ within $30 \mathrm{~s}$ before reaching equilibrium for $3 \mathrm{~min}$. The injection volume was $2 \mu \mathrm{L}$ and the column was maintained at $35^{\circ} \mathrm{C}$. All samples were injected twice. The mass spectrometer was operated in heated electrospray ionization (HESI) mode with a spray voltage of $3.5 \mathrm{kV}$, a capillary temperature of $300^{\circ} \mathrm{C}$, a sheath gas flow of 50 , an assist gas of 10.S lens RF level set at 40 and an S lens voltage set at $25 \mathrm{~V}$. The collecting resolution ratio of full scan was 70,000 and the MS/MS collecting list was 17,500.

\section{The identification and screening of the metabolites}

The Pareto-scaling method was used for normalization. Pareto-scaling divides the peak area of each individual component in the data matrix by the square root of the standard deviation of the peak area of the component in the variable set and compensates for the large range of responses between the variables. ${ }^{19,20}$ The differences analysis and enrichment analysis were performed simultaneously by using MetaboAnalyst 4.0 software and XCMS software. This study applied the Fold Change Analysis (FC Analysis), univariate statistical analysis of metabolites by $t$-test (The $t$-test was used for comparison between the two groups) and the drawing of Volcano Plot. The screening criteria for differential metabolic indicators include $p<0.05$, fold change $>2$ or $<0.5$. The differential data were analyzed by Metlin_AMRT_PCDL and Metlin_Lipids_AM_PCDL database to identify the corresponding metabolites. Then by using the metabolites with significant differences to conduct hierarchical clustering of the samples of different groups. The potential biomarkers that could predict cervical cancer and precancerous lesions were screened out by using the clustering of the samples from different groups and expression patterns of different 
metabolites that were analyzed by hierarchical clustering.

\section{Data analysis}

The differential metabolites were submitted to KEGG online database and the XCMS-Online Website to be analyzed simultaneously. The differential metabolites were screened out by using chemometrics and searched in the HMDB and KEGG online database to perform the qualitative analysis and metabolic pathway analysis.

\section{Statistical analysis}

The data were analyzed by SPSS20.0 software. The results of the normal distribution of the measurement data were expressed as mean \pm standard deviation. The $t$-test was used for comparison between the two groups. One-way analysis of variance was used for comparison between the two groups. $p<0.05$ was considered to be statistically significant.

\section{Results}

The diagnostic information of the

\section{laboratory serology}

The laboratory serological information had included the age, ALT, AST, TP, ALB, Urea, Crea, AFP, CEA, CA125, CA153 and CA199 of the subjects. From the current diagnostic data of cervical cancer, it is hard to differentiate various stages of the disease in terms of precancerous cervical lesions by using AFP, CEA,
CA125, CA153 and CA199. Therefore, it is of great significance to find out new serum diagnostic biomarkers (Table 1).

\section{Statistical analysis of incorporated group sample and differential metabolite distribution}

As presented in Figure 1, we examined the serum metabolic function of patients with cervical inflammation, cervical cancer and precancerous lesions by LC/MS. Prior to the formal analysis of the SMICA-P13.0 software, the data sets were normalized to obtain more intuitive and reliable results. In order to determine whether there were differences among the five groups (healthy people, cervicitis patients, HSIL patients, LSIL patients and CC patients), we used PCA and OPLS-DA modeling to analyze the samples. The OPLS-DA principal component analysis of the three groups showed that the three components were well grouped, with a high association within the group and the large differences among the groups, especially for the three groups of cervical cancer patients, healthy people and precancerous lesions.

As shown in Figure 2, in order to further screen out different substances between different groups, a univariate statistical analysis of metabolites was performed using Fold Change Analysis (FC Analysis) and $t$-test, and a volcano map was drawn (Volcano Plot), with $p$ value $<0.05$, fold change $>2$ times or $<0.5$ as screening criteria for differential metabolic indicators. By screening, we

Table I The serological diagnostic information of all samples in the laboratory

\begin{tabular}{|c|c|c|c|c|c|c|}
\hline & CON & IF & LSIL & HSIL & CC & TREAT \\
\hline Number of subjects & 11 & 10 & 5 & 8 & 19 & 14 \\
\hline Age & $45.82 \pm 7.92$ & $42 \pm 11.37$ & $38.8 \pm 9.524$ & $50.25 \pm 9.30$ & $50.63 \pm 8.694$ & $55.14 \pm 7.79$ \\
\hline HPV ( \pm$)$ & - & + & + & + & + & + \\
\hline ALT (U/L) & $18.55 \pm 15.64$ & $|3.1| \pm 5.06$ & $22.8 \pm 8.96$ & $13.5 \pm 3.46$ & $17.58 \pm 10.15$ & $27.43 \pm 32.60$ \\
\hline AST (U/L) & $19.27 \pm 10.97$ & $16.7 \pm 2.83$ & $19.2 \pm 4.44$ & $15.88 \pm 1.25$ & $|8.2| \pm 4.44$ & $23.86 \pm 10.60$ \\
\hline TP $(g / L)$ & $68.33 \pm 7.22$ & $62.8 \pm 14.79$ & $66.72 \pm 2.68$ & $69.28 \pm 5.41$ & $68.96 \pm 5.02$ & $60.84 \pm 5.45$ \\
\hline ALB (g/L) & $41.85 \pm 3.37$ & $41.47 \pm 2.82$ & $41.84 \pm 3.53$ & $43.13 \pm 2.50$ & $40.59 \pm 4.96$ & $36.9 \pm 3.4 I$ \\
\hline Urea $(\mathrm{mmol} / \mathrm{L})$ & $4.63 \pm 2.77$ & $4.30 \pm 0.89$ & $4.29 \pm 1.24$ & $4.4 I \pm 0.88$ & $4.39 \pm 1.26$ & $3.85 \pm 2.35$ \\
\hline Crea $(\mu \mathrm{mol} / \mathrm{L})$ & $60.36 \pm 15.47$ & $64.3 \pm 4.95$ & $54 \pm 7.87$ & $54.75 \pm 7.87$ & $59.74 \pm 12.57$ & $61.57 \pm 8.06$ \\
\hline $\operatorname{AFP}(\mathrm{ng} / \mathrm{mL})$ & $1.92 \pm 0.6 \mathrm{I}$ & $2.10 \pm 0.62$ & $2.08 \pm 0.78$ & $3.28 \pm 2.28$ & $2.63 \pm 1.00$ & $2.92 \pm 1.28$ \\
\hline CEA (ng/mL) & $1.65 \pm 1.22$ & $1.78 \pm 0.75$ & $1.85 \pm 1.61$ & $1.61 \pm 0.85$ & $15.09 \pm 53.84$ & $1.74 \pm 1.27 \mid$ \\
\hline CAI25 (U/mL) & $15.47 \pm 9.32$ & $16.38 \pm 7.45$ & $12.58 \pm 4.69$ & $26.5 \pm 44.31$ & $23.18 \pm 24.25$ & $68.67 \pm 195.3$ \\
\hline CAI53 (U/mL) & $|2.86 \pm 6.6|$ & $9.34 \pm 5.3$ & $12.63 \pm 6.30$ & $13.63 \pm 6.60$ & $14.97 \pm 19.14$ & $12.22 \pm 9.23$ \\
\hline CAI99 (U/mL) & $8.46 \pm 5.16$ & $8.46 \pm 5.16$ & $16.7 \pm 10.36$ & $15.8| \pm 1| .89$ & $18.85 \pm 27.75$ & $|4.79 \pm 8.6|$ \\
\hline
\end{tabular}

Abbreviations: CON, control; IF, inflammation; ISIL, low grade squamous intraepithelial lesion; HSIL, high grade squamous intraepithelial lesion; CC, cervical cancer. 

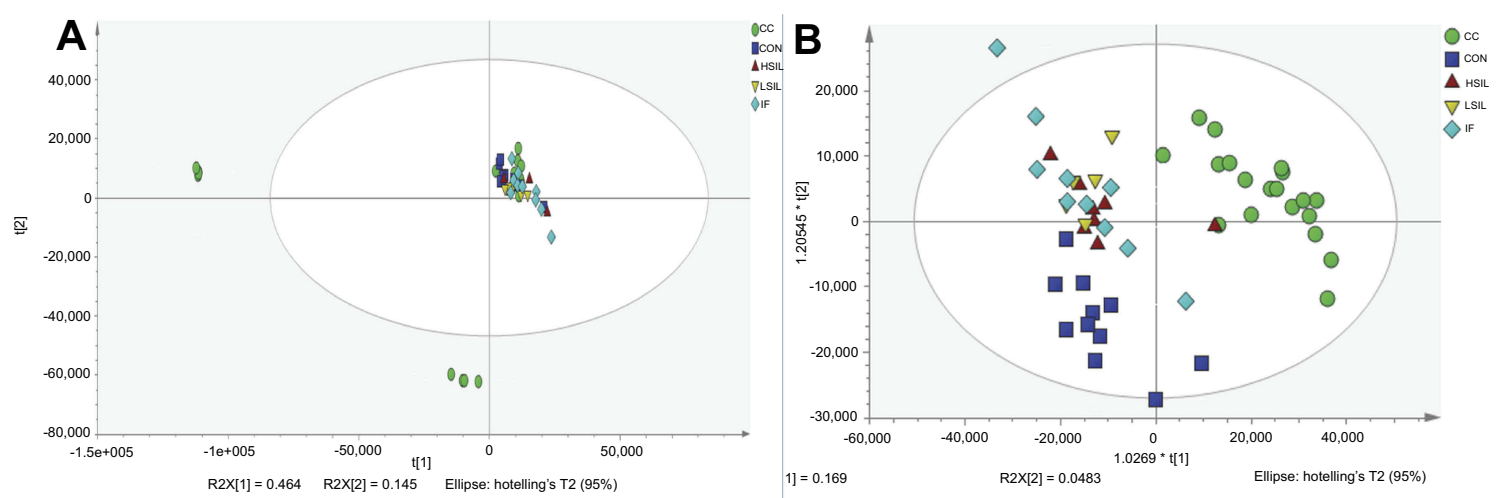

Figure I PCA analysis and OPLS-DA analysis of serum metabolites in each group of patients. (A) PCA analysis of CON, IF, LSIL, HSIL and CC groups. (B) PLS-DA analysis of CON, IF, LSIL, HSIL and CC groups.

A



C

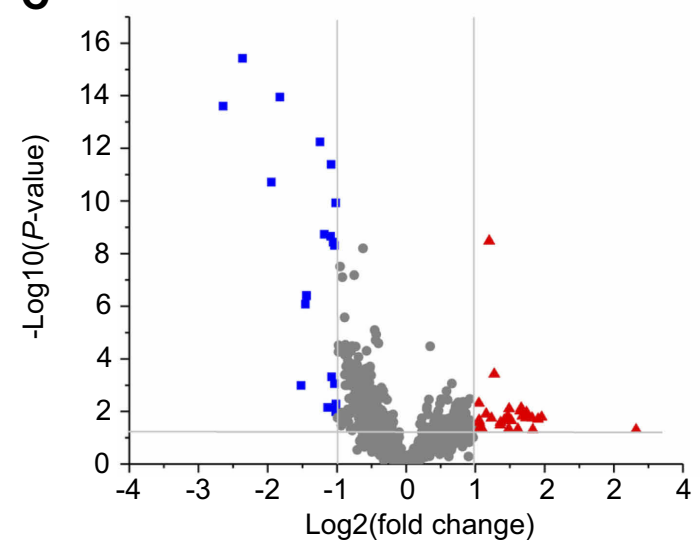

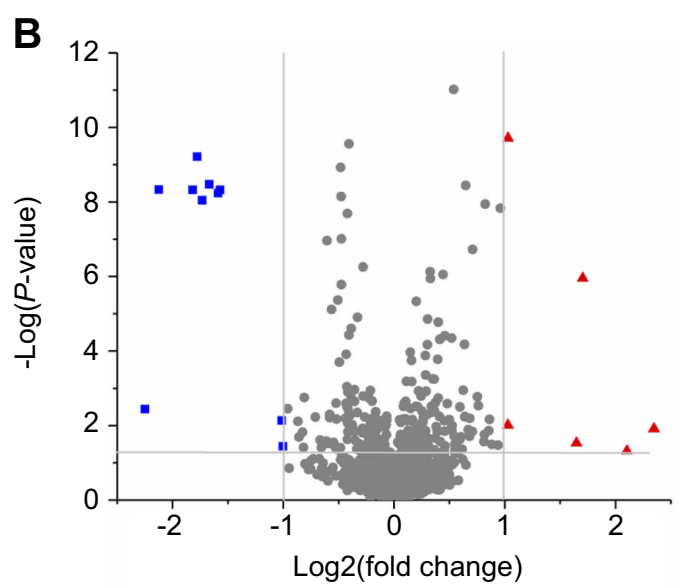

D

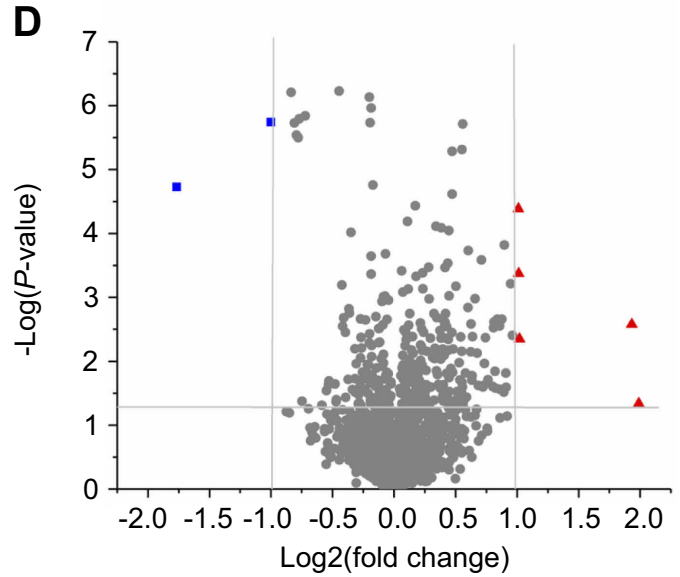

Figure 2 Volcano Plot of serum metabolites in each group of patients. (A) Volcanic map of serum metabolites in LSIL patients compared to healthy individuals. (B) Volcanic map of serum metabolites in HSIL patients compared to healthy individuals. (C) Volcanic map of serum metabolites in patients with CC compared to healthy subjects. (D) Volcanic map of serum metabolites in patients with cervical IF compared to healthy subjects. Red dots were significantly up-regulated metabolites (FC $>2$, $p<0.05$ ), and blue dots were significantly down-regulated metabolites ( $F C<0.5, p<0.05)$.

Abbreviations: CON, control; IF, inflammation; ISIL, low grade squamous intraepithelial lesion; HSIL, high grade squamous intraepithelial lesion; CC, cervical cancer; FC, fold change analysis.

found out that compared to the control group, the LSIL group had 14 differentiate metabolites, in which 5 of them were significantly up-regulated and 10 significantly down- regulated; the HSIL group had 16 differentiate metabolites, in which 6 of them were significantly up-regulated and 10 significantly down-regulated; the $\mathrm{CC}$ group had 49 
Table 2 Compared with healthy people, cervical precancerous lesions - screening results of serum metabolomics differences in patients with LSIL

\begin{tabular}{|c|c|c|}
\hline Metabolite name & FC & raw. pval \\
\hline 6alpha-Fluoro-I I beta, I7-dihydroxypregn-4-ene-3,20-dione & 13.20 & $4.46 \times 10^{5}$ \\
\hline PA $(O-20: 0 / 17: 2(9 Z, \mid 12 Z))$ & 2.37 & 0.0061 \\
\hline PE (I6:I(9Z)/P-I8:I(IIZ)) & 2.16 & 0.0153 \\
\hline Ala Cys Ser Trp & 2.06 & 0.0092 \\
\hline Pro Glu Arg Val Lys & 0.45 & 0.0047 \\
\hline Dodecanoylcarnitine & 0.45 & $5.00 \times 10^{5}$ \\
\hline (3R)-3-isopropenyl-6-oxoheptanoic acid & 0.34 & 0.0300 \\
\hline Tomatidine & 0.32 & $3.11 \times 10^{6}$ \\
\hline $\begin{array}{l}\text { I-[(2S)-2-[[(2S)-2-(2,4-dioxo-IH-quinazolin-3-yl)-3-phenylpropanoyl] amino]-3-methylpentanoyl] piperidine-4-carboxylic } \\
\text { acid }\end{array}$ & 0.11 & 0.0132 \\
\hline
\end{tabular}

Abbreviation: FC, old change analysis.

Table 3 Compared with healthy people, cervical precancerous lesions - screening results of serum metabolomics differences in patients with HSIL

\begin{tabular}{|c|c|c|}
\hline Metabolite name & FC & raw. pval \\
\hline 6alpha-Fluoro-I I beta, I7-dihydroxypregn-4-ene-3,20-dione & 15.36 & $8.04 \times 10^{5}$ \\
\hline L-Carnitine & 4.20 & 0.0468 \\
\hline Ala Cys Ser Trp & 3.26 & $1.11 \times 10^{6}$ \\
\hline 2-Amino-3-methyl-I-butanol & 3.13 & 0.0293 \\
\hline Choline & 2.89 & 0.0282 \\
\hline $\begin{array}{l}\text { I-[(2S)-2-[[(2S)-2-(2,4-dioxo-IH-quinazolin-3-yl)-3-phenylpropanoyl] amino]-3-methylpentanoyl] piperidine-4-carboxylic } \\
\text { acid }\end{array}$ & 0.42 & 0.0340 \\
\hline Soladulcidine & 0.23 & $4.65 \times 10^{9}$ \\
\hline
\end{tabular}

Abbreviation: FC, old change analysis.

Table 4 Compared with healthy people, cervical cancer - screening results of serum metabolomics differences in patients with CC

\begin{tabular}{|c|c|c|}
\hline Metabolite name & FC & raw. pval \\
\hline 6alpha-Fluoro-I I beta, 17-dihydroxypregn-4-ene-3,20-dione & 11.84 & 0.0037 \\
\hline Penaresidin A/B & 10.00 & 0.0495 \\
\hline Asn Asn Gln Arg & 3.29 & 0.0147 \\
\hline 3-O-L-rhamnosyl-3-hydroxydecanoyl-3-hydroxydecanoic acid & 3.17 & 0.0074 \\
\hline Phe lle Met Val & 2.86 & 0.0220 \\
\hline Ala lle Gln Arg & 2.79 & 0.0452 \\
\hline Ala Cys Ser Trp & 2.42 & 0.0004 \\
\hline Prosopinine & 2.35 & 0.0179 \\
\hline 2-amino-|4,16-dimethyloctadecan-3-ol & 2.30 & $3.33 \times 10^{9}$ \\
\hline $\begin{array}{l}\text { I-[(2S)-2-[[(2S)-2-(2,4-dioxo-IH-quinazolin-3-yl)-3-phenylpropanoyl] amino]-3-methylpentanoyl] piperidine-4-carboxylic } \\
\text { acid }\end{array}$ & 0.42 & 0.0071 \\
\hline 3'-O-Aminopropyl-25-hydroxyvitamin & 0.42 & $5.75 \times 10^{13}$ \\
\hline Soladulcidine & 0.16 & $2.48 \times 10^{14}$ \\
\hline
\end{tabular}

Abbreviation: FC, old change analysis.

differentiate metabolites, in which 29 of them were significantly up-regulated and 20 significantly downregulated; the IF group had 7 differentiate metabolites, in which 5 of them were significantly up-regulated and 2 significantly down-regulated.

\section{Differentiate plasma metabolites in different groups}

Through database comparison, we found that compared with the CON group, we screened out 9 metabolites with 
Table 5 Compared with healthy people, Inflammation - screening results of serum metabolomics differences in patients with IF

\begin{tabular}{|c|c|c|}
\hline Metabolite name & FC & raw. pval \\
\hline PE (I8:I(IIZ)/P-I8:I(IIZ)) I6:0) & 2.41 & 0.0007 \\
\hline PC (O-I6:0/I8:3(6Z,9Z, I2Z)) & 2.09 & 0.0002 \\
\hline PA (O-20:0/I7:2(9Z,I2Z)) & 2.08 & 0.0125 \\
\hline PS (O-16:0/20:2(IIZ,I4Z)) & 2.06 & 0.0032 \\
\hline Ala Cys Ser Trp & 2.01 & $4.10 \times 10^{5}$ \\
\hline Soladulcidine & 0.50 & $1.80 \times 10^{6}$ \\
\hline 4S-hydroxylauric acid & 0.35 & 0.0169 \\
\hline I-[(2S)-2-[[(2S)-2-(2,4-dioxo-| H-quinazolin-3-yl)-3-phenylpropanoyl] amino]-3-methylpentanoyl] piperidine-4-carboxylic acid & 0.36 & 0.0172 \\
\hline
\end{tabular}

Abbreviation: FC, old change analysis.

differential expression in the LSIL group, 4 of which were up-regulated and 5 were down-regulated (Table 2); 7 metabolites with differential expression were screened out in the HSIL group screened, in which 5 of them were up-regulated and 2 were down-regulated (Table 3); 12 matabolites with differential expression were screened out in the CC group, in which 9 were up-regulated and 3 were down-regulated (Table 4); 8 metabolites with differential expression were screened in IF group, in which 5 were up-regulated and 3 were down-regulated (Table 5).

Through the comparison of different substances between groups, we found that Dodecanoylcarnitine, (3R)-3-isopropenyl-6-oxoheptanoic acid and Tomatidine could be used as potential biomarkers for distinguishing LSIL patients; L-Carnitine, 2-Amino-3-methyl-1- Butanol and Choline could be used as potential biomarkers for distinguishing patients with HSIL; Penaresidin A/B, Asn Asn Gln Arg, 3-OL-rhamnosyl-3-hydroxydecanoyl3-hydroxydecanoic acid, Phe Ile Met Val, Ala Ile Gln Arg, Prosopinine, 2-amino-14,16-dimethyloctadecan-3-ol and 3'-O-Aminopropyl-25-hydroxyvitamin could be used as potential biomarkers for distinguishing patients with CC. PC, PS and 4S-hydroxylauric acid could be used as a potential biomarker for distinguishing cervical IF patients.

\section{Cluster analysis and identification of serum metabolic biomarkers}

The cluster analysis of the differential metabolites of patients in groups of healthy people, cervicitis, LSIL, HSIL, CC and Treatment (Figure 3). We found that in the CON, LSIL and HSIL groups, 2-Amino-3-methyl1-butanol, L-Carnitine, Asn Asn Gln Arg, and Ala Cys Ser Trp were continuously up-regulated, and soladulcidine and Ala Ile Gln Arg were continuously down-regulated.
2-Amino-3-methyl-1-butanol, L-Carnitine, Asn Asn Gln Arg, Ala Cys Ser Trp, soladulcidine, Ala Ile Gln Arg can be used as potential biomarkers for predicting different stages of cervical precancerous lesions (Figure 4A-F). Prosopinine was continuously up-regulated in the $\mathrm{CON}$, IF, LSIL, HSIL, and CC groups, and was initially downregulated in the TREAT group. Prosopinine was expected to serve as a potential marker for different stages of cervical cancer and precancerous lesions (Figure 4G). Interestingly, the expression of 17-dihydroxypregn-4-ene3,20-dione in cervical cancer and precancerous lesions was higher than that in CON group 6alpha-Fluoro-11beta, and it was significantly reduced in the TREAT group, indicating that it could serve as a potential biomarker for assessing the prognosis of cervical cancer (Figure 4H).

\section{The function analysis of differentiate metabolites}

Pathological enrichment analysis was performed on Metabolites with $p$ values $<0.05$, fold change greater than 2-fold or less than 0.05 -fold by applying MetaboAnalyst 4.0. The results are shown in Figure 5.

The results showed that the differentially expressed metabolites mainly participated in the biological metabolic processes of phosphatidylethanolamine biosynthesis, phosphatidylcholine biosynthesis, pantothenate and CoA biosynthesis, mitochondrial beta-oxidation of short-chain saturated fatty acids, mitochondrial beta-oxidation of longchain saturated fatty acids and sphingolipid metabolism.

\section{Discussion}

The development of cancer is often accompanied by complex host systemic responses, usually reflected in plasma proteins and metabolites, so plasma proteins and metabolites are highly likely to contain cancer marker information. 


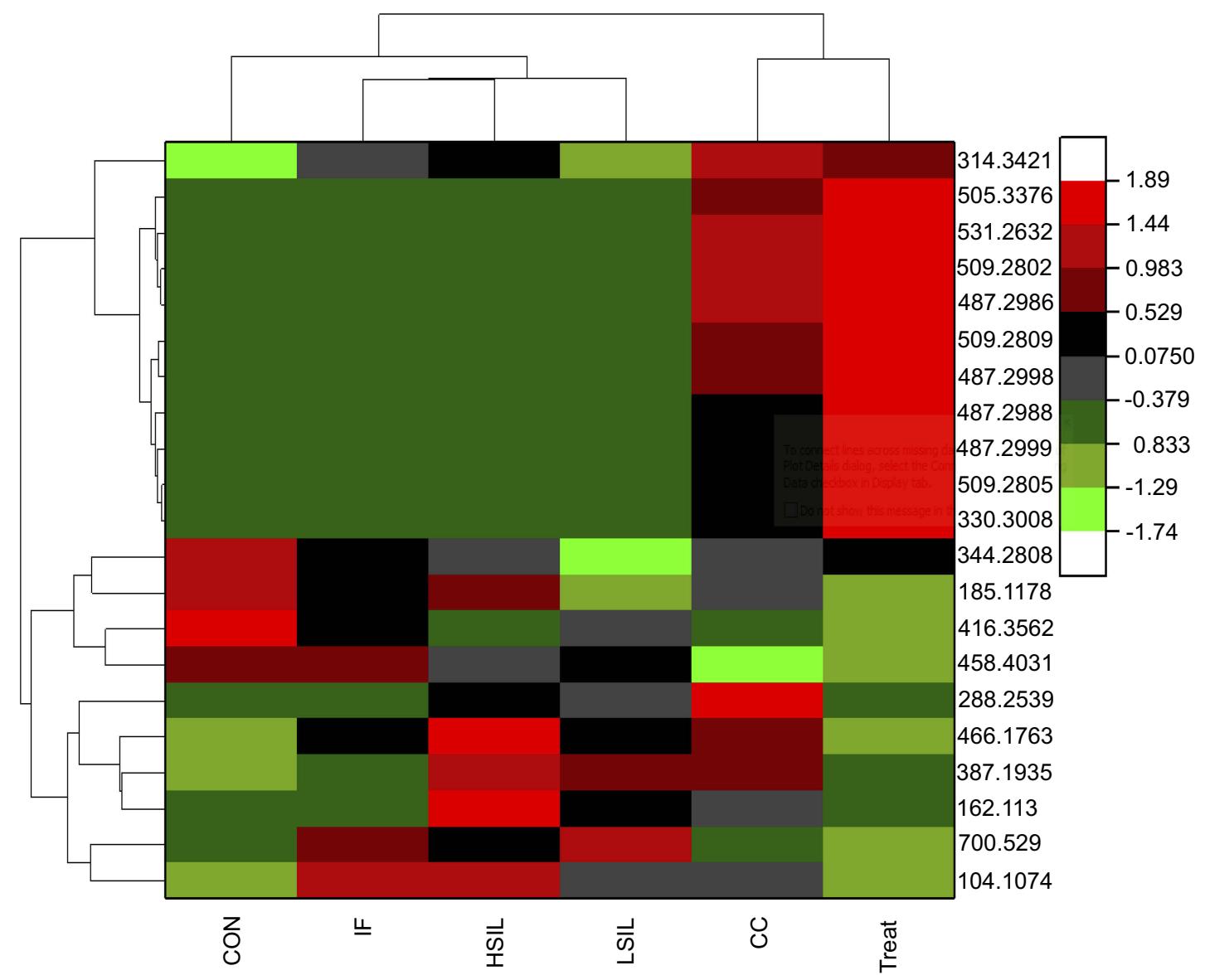

Figure 3 Heat map cluster analysis of potential serum metabolic biomarkers in patients with cervical cancer, precancerous lesions and cervicitis. The abscissa is the sample grouping and the ordinate is the $\mathrm{M} / \mathrm{z}$ of the metabolite.

Abbreviations: CON, control; IF, inflammation; ISIL, low grade squamous intraepithelial lesion; HSIL, high grade squamous intraepithelial lesion; CC, cervical cancer.

However, there are few new types of cancer metabolic biomarkers that can be applied to clinical practice, so it is an urgent task to conduct fundamental research. At present, studies on serum and urine metabolic markers of cervical cancer have been reported, ${ }^{15-17}$ but few studies on serum metabolic markers of cervical precancerous lesions have been reported. ${ }^{21}$ Due to the diversity and specificity of the serum, experiments and setting parameters of metabolomics data make differences in the screening of metabolic markers. ${ }^{21}$ To the best of our knowledge, there have been no reports of serum metabolomics studies on cervical cancer and precancerous lesions in southern China. ${ }^{14,22}$

Therefore, this study aims to identify the serum metabolic profiling of patients with cervical canal inflammation, LSIL, HSIL and CC in the population of southern China in Guangdong by LC-MS technology. The potential serum metabolic biomarkers for cervical precancerous diagnosis and CC monitoring were screened out by comparing serum metabolites in healthy people. Several substances may play a role as biological markers in CC, such as the carcinoembryonic antigen, some proteins, amino acids, enzymes and their metabolites as well as serum. ${ }^{23}$ By comparing serum metabolic data between cervical cancer treatment group and healthy people, serum metabolic markers that could be applied to the evaluation of cervical cancer treatment efficacy were identified. The study found that Dodecanoyl carnitine and (3R)-3-isopropenyl-6-oxo heptanoic acid could serve as a potential serum metabolism biomarker for LSIL patients; L-Carnitine, 2-Amino-3-methyl-1-butanol and Choline could serve as a biomarker for differential serum metabolism in patients with HSIL, Penaresidin A/B, Asn Asn Gln Arg, 3-OL-rhamnosyl-3-hydroxydecanoyl-3-hydroxydecanoic acid, Phe Ile Met Val, AlaIleGln Arg, Prosopinine, 2-amino-14,16-Dimethyloctadecan-3-ol and 3'-O-Amino-propyl-25-hydroxyvitamin could serve as a potential biomarker of serum metabolism in patients with CC; PC, PS and 4S-hydroxylauric acid could serve as a potential biomarker for distinguishing cervical IF patients. 

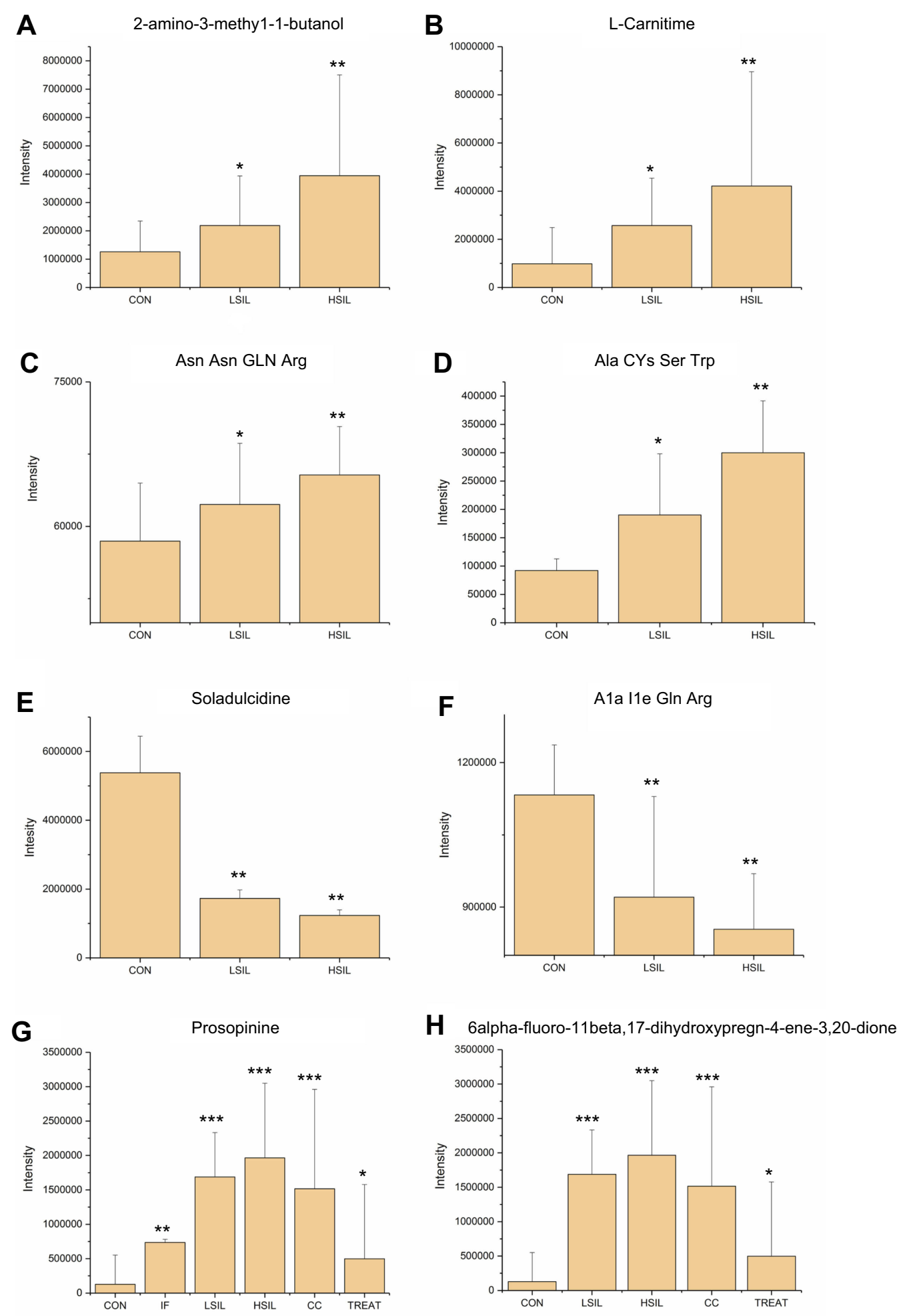

Figure 4 Analysis of serum metabolic biomarkers in patients from each group. (A) 2-Amino-3-methyl-I-butanol was continuously up-regulated in the LSIL, HSIL group compared with CON. (B) L-Carnitine is continuously up-regulated in the LSIL and HSIL groups compared with CON. (C) Asn Asn Gln Arg is continuously up-regulated in the LSIL and HSIL groups compared with CON. (D) Ala Cys Ser Trp is continuously up-regulated in the LSIL, HSIL group compared with CON. (E) Soladulcidine was continuously down-regulated in the LSIL and HSIL groups compared with CON. (F) Ala lle Gln Arg is continuously down-regulated in the LSIL and HSIL groups compared with CON. (G) Prosopinine was continuously up-regulated in the CON, IF, LSIL, HSIL and CC groups and began to be lowered in the TREAT group. (H) 6alpha-Fluoro - I l beta, 17-dihydroxypregn-4-ene-3, 20-dione was higher in the cervical cancer and precancerous lesions than in the CON group and significantly decreased in the TREAT group. $* p<0.05, * * p<0.01$, $* * * p<0.001$ vs Con group.

Abbreviations: CON, control; IF, inflammation; ISIL, low grade squamous intraepithelial lesion; HSIL, high grade squamous intraepithelial lesion; CC, cervical cancer. 
A

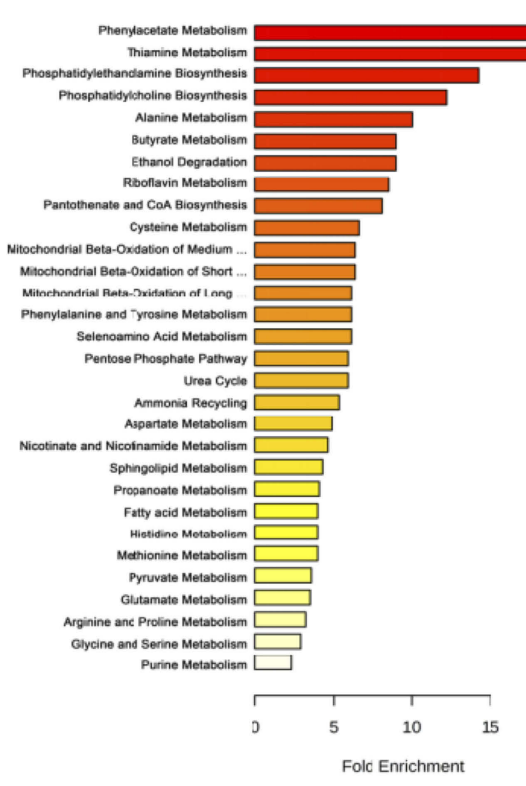

B

Metabolite Sets Enrichment Overview

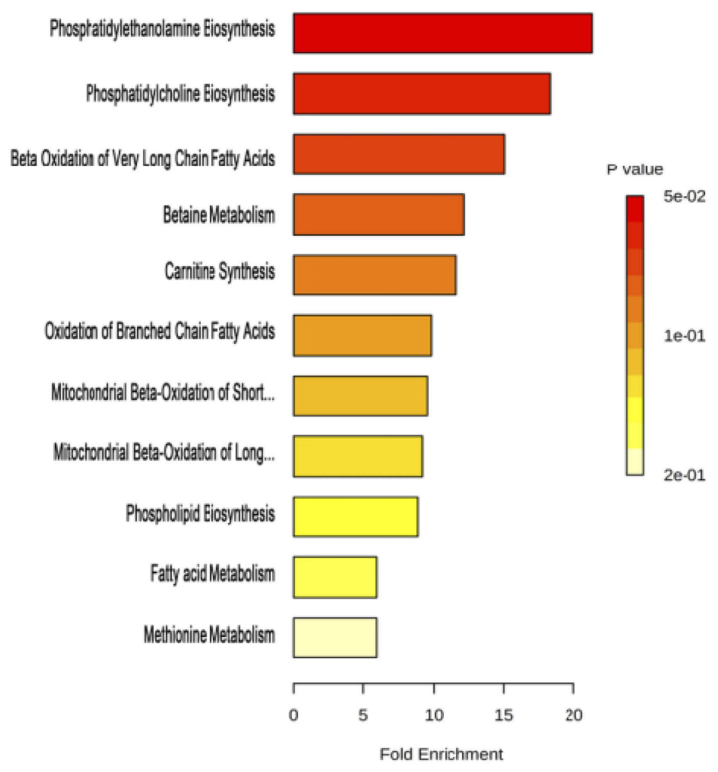

C



Metabolite Sets Enrichment Overview

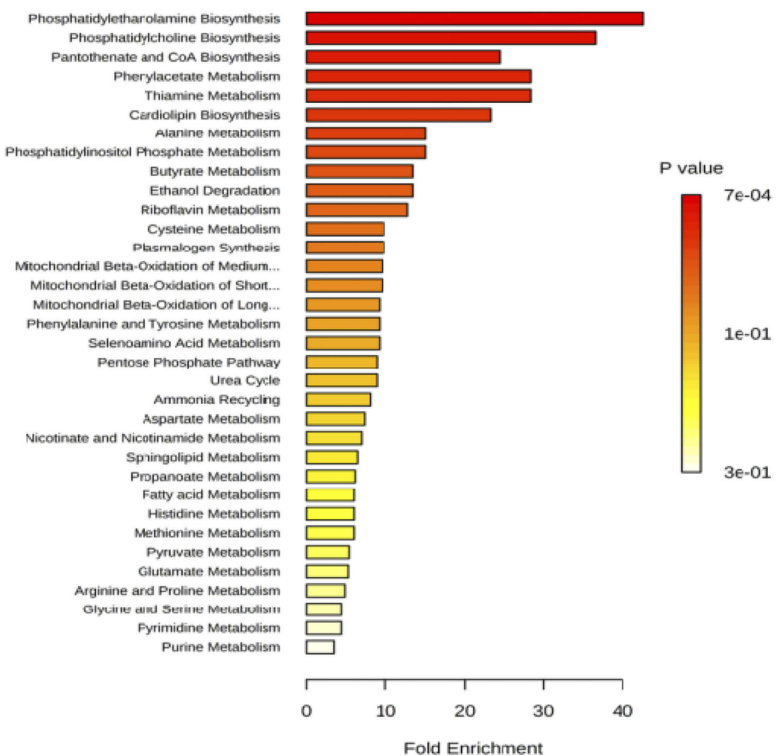

Figure 5 Differential metabolic material enrichment analysis results. (A) Enrichment analysis of differential metabolites in healthy people with LSIL. The results showed that the differential metabolites were mainly enriched in Phenylacetate Metabolism, Thiamine Metabolism, Phosphatidylethanolamine Biosynthesis and Phosphatidylcholine Biosynthesis. (B) Enrichment analysis of differential metabolites in healthy people with HSIL. The results showed that the differential metabolites were mainly enriched in Phosphatidylethanolamine Biosynthesis, Phosphatidylcholine Biosynthesis, Beta Oxidation of Very Long-Chain Fatty Acids, Betaine Metabolism and Carnitine Synthesis. (C) Enrichment analysis of differential metabolites in healthy people with cervical inflammation showed that the differential metabolites were mainly enriched in Phosphatidylethanolamine Biosynthesis, Phosphatidylcholine Biosynthesis, Pantothenate and CoA Biosynthesis, Phenylacetate Metabolism and Thiamine Metabolism. Abbreviations: CON, control; IF, inflammation; ISIL, low grade squamous intraepithelial lesion; HSIL, high grade squamous intraepithelial lesion; CC, cervical cancer.

More importantly, the volume of 2-Amino-3-methyl1-butanol, L-Carnitine, Asn Asn Gln Arg and Ala Cys Ser Trp gradually increased in serum of healthy people, HSIL and LSIL groups; the volume of soladulcidine and Ala Ile Gln Arg gradually decreased in serum of healthy people, HSIL and LSIL groups. In general, these six substances can be used as the potential serum metabolic biomarkers to predict the precancerous lesions of cervical cancer in different stages. Besides, the metabolic Prosopinine gradually increased in the healthy population, patients with LSIL, cervical tuberculosis patients, patients with HSIL and cervical cancer patients, and the content 
of this metabolite in the cervical cancer treatment improvement group decreased significantly. It is likely that Prosopinine can serve as a potential serum metabolic marker for different stages of cervical cancer, cervical inflammation and precancerous lesions. Further functional enrichment analysis revealed that these potential serum metabolite biomarkers were mainly involved in biometabolism process including phosphatidylethanolamine biosynthesis, phosphatidylcholine biosynthesis, pantothenate and $\mathrm{CoA}$ biosynthesis, mitochondrial beta-oxidation of short-chain saturated fatty acids, mitochondrial betaoxidation of long-chain saturated fatty acids,and sphingolipid metabolism, which were closely related to the body's protein response such as stress response, inflammation induction, immune regulation, cell transfer and coagulation cascade.

\section{Conclusion}

In general, by LC-MS/MS and proteomics analysis, the study has screened out 7 new types of serum metabolites that can be used as the diagnostic markers of the treatment efficacy of precancerous lesions of cervical cancer and the level of the canceration. Considering the importance of the oligopeptide in the body's metabolism, it is of great significance to notice the huge difference of the two polypeptides (Asn Asn Gln Arg and Ala Cys Ser Trp) in the serum metabolism of the patients, and the Prosopinine can serve as a potential serum metabolic marker for different stages of cervical cancer, cervical inflammation and precancerous lesions. More importantly, the role and function of these metabolites in the happening and development of cervical cancer have not been reported yet, but the related mechanisms of the immune, inflammatory, metastatic and coagulation cascade pathways deserve further investigation.

\section{Acknowledgments}

This work was supported by the Medical and Health Research Project of Foshan City (20190282); Medical Science and Technology Research Project of Foshan City (2018AB001112); Medical Key Discipline Planning Project of Foshan City (FSZDZK135045), and Science and Technology Planning Project of Guangdong Province (2019B020208009).

\section{Disclosure}

The authors report no conflicts of interest in this work.

\section{References}

1. Ferlay J, Soerjomataram I, Ervik M, et al. Cancer incidence and mortality worldwide: IARC CancerBase. Abdom Radiol (NY). 2012;42(1):124-140. doi:10.1007/s00261-016-0901-x

2. Chen W, Zheng R, Baade PD, et al. Cancer statistics in China, 2015. CA Cancer J Clin. 2016;66(2):115-132. doi:10.3322/caac.21338

3. Schiffman M, Castle PE. Human papillomavirus: epidemiology and public health. Arch Pathol Lab Med. 2003;127(8):930-934. doi:10.1043/1543-2165(2003)127<930:HPEAPH>2.0.CO;2

4. Wright TC Jr. HPV DNA testing for cervical cancer screening. FIGO 26th Annual Report on the Results of Treatment in Gynecological Cancer. Int $J$ Gynaecol Obstet. 2006;95 Suppl:1S239-S246. doi:10.1016/S0020-7292(06)60039-8

5. Wilting SM, Smeets SJ, Snijders PJ, et al. Genomic profiling identifies common HPV-associated chromosomal alterations in squamous cell carcinomas of cervix and head and neck. BMC Med Genomics. 2009;2(1):32-43. doi:10.1186/1755-8794-2-32

6. Stoler MH, Vichnin M, Ferenczy A, et al. The accuracy of colposcopic biopsy: analyses from the placebo arm of the Gardasil clinical trials. Int J Cancer. 2011;128(6):1354-1362. doi:10.1002/ ijc. 25470

7. Wentzensen N, Fetterman B, Castle PE, et al. p16/Ki-67 dual stain cytology for detection of cervical precancer in HPV-positive women. J Natl Cancer Inst. 2015;107(12):1-8. doi:10.1093/jnci/djv257

8. He XK, Luo XP, Mao LZ, et al. An optoelectronic cervical cancer screening system for screening cervical cancer: comparison with cervical cytology. Nan Fang Yi Ke Da Xue Xue Bao. 2010;30 (10):2304-2306.

9. Xin-Chun LI, Shang JB, Xiao-Mei WU, et al. MRI findings of uterine cervical cancer and value of MRI in preoperative staging. Nan Fang Yi Ke Da Хие Хие Bao. 2007;27(3):352-354.

10. Ballesta AM, Torre GC, Bombardieri E, et al. Up dating on tumor markers in tissues and in biological fluids. Minerva Med. 1993;44 (2):75-78.

11. Angiolo G, Stefania C, Angelo C, et al. Serum tumor markers in the management of ovarian, endometrial and cervical cancer. Biomed Pharmacother. 2004;58(1):24-38. doi:10.1016/j.biopha.2003. 11.003

12. Peiyuan Y, Guowang X. Metabolomics for tumor marker discovery and identification based on chromatography-mass spectrometry. Expert Rev Mol Diagn. 2013;13(4):339-348. doi:10.1586/erm.13.23

13. Nicholson JK, Lindon JC. Systems biology: metabonomics. Nature. 2008;455(7216):1054-1056. doi:10.1038/4551054a

14. Huang S, Chong N, Lewis NE, et al. Sialic acid metabolism: a key player in breast cancer metastasis revealed by metabolomics. Front Oncol. 2018:8174-8189. doi:10.3389/fonc.2018.00174

15. Yang K, Xia B, Wang W, et al. A comprehensive analysis of metabolomics and transcriptomics in cervical cancer. Sci Rep. 2017;7 (1):43353-43364. doi:10.1038/srep43353

16. Ye N, Liu C, Shi P. Metabolomics analysis of cervical cancer, cervical intraepithelial neoplasia and chronic cervicitis by $1 \mathrm{H}$ NMR spectroscopy. Eur J Gynaecol Oncol. 2015;36(2):174-180.

17. Hasim A, Ali M, Mamtimin B, Ma J-Q, Li Q-Z, Abudula A. Metabonomic signature analysis of cervical carcinoma and precancerous lesions in women by $1 \mathrm{H}$ NMR spectroscopy. Exp Ther Med. 2012;3(6):945-951. doi:10.3892/etm.2012.509

18. Liang Q, Yu Q, Wu H, Zhu YZ, Zhang AH. Metabolite fingerprint analysis of cervical cancer using LC-QTOF/MS and multivariate data analysis. Anal Methods. 2014;6(12):3937-3942. doi:10.1039/ C4AY00399C

19. Ramadan Z, Jacobs D, Grigorov M, Kochhar S. Metabolic profiling using principal component analysis, discriminant partial least squares, and genetic algorithms. Talanta. 2006;68(5):1683-1691. doi:10.1016/j.talanta.2005.08.042 
20. Weljie AM, Jack N, Pascal M, et al. Targeted profiling: quantitative analysis of $1 \mathrm{H}$ NMR metabolomics data. Anal Chem. 2006;78 (13):4430-4442. doi:10.1021/ac060209g

21. Huang S, Chong N, Lewis NE, Jia W, Xie G, Garmire LX. Novel personalized pathway-based metabolomics models reveal key metabolic pathways for breast cancer diagnosis. Genome Med. 2016;8(1):34-48. doi:10.1186/s13073-0160289-9
22. Singh S, Eaton JE, Murad MH, Tanaka H, Iijima H, Talwalkar JA. Accuracy of spleen stiffness measurement in detection of esophageal varices in patients with chronic liver disease: systematic review and meta-analysis. Clin Gastroenterol Hepatol. 2014;12(6):935-945. doi:10.1016/j.cgh.2013.09.013

23. Oehr P, Adolphs HD. [Significance of biological markers for the diagnosis of urinary bladder cancer]. Onkologie. 1980;3(5):238-246. doi: $10.1159 / 000214801$

\section{Publish your work in this journal}

Cancer Management and Research is an international, peer-reviewed open access journal focusing on cancer research and the optimal use of preventative and integrated treatment interventions to achieve improved outcomes, enhanced survival and quality of life for the cancer patient.
The manuscript management system is completely online and includes a very quick and fair peer-review system, which is all easy to use. Visit http://www.dovepress.com/testimonials.php to read real quotes from published authors. 